\title{
Mechanics Analysis of Overhead Transmission Lines Based On-line Monitoring
}

\author{
Lili Dai, Yongli Zhu, Zehui Liang \\ School of Electrical and Electronic Engineering, North China Electric Power University, Baoding,China \\ Email: lilidai070582@126.com
}

Received 2013

\begin{abstract}
At present, the on-line monitoring is widely applied to the power line monitoring. In this paper, a new mechanical calculation model is established according to the on-line monitoring. And this model is based on the parameters that tension sensors and angle sensors on suspended points detect, and combines with the parameters of the wire itself, and also considers the deflection angel of wires due to wind. In this model, mechanics parameters of wires are turned into the new coordinate plane after deflection angel of wires due to wind, or windage yaw plane. A statics tension balance equation is built in the vertical direction of the new windage yaw plane. According to the theoretical analysis and algorithm, we verify the accuracy of this newly developed mechanical calculation model.
\end{abstract}

Keywords: Overhead Transmission Line; On-line Monitoring; Mechanical Analysis; Tension; Angle; Icing; Windage Yaw

\section{Introduction}

Affected by climate, macrophotography and meteorological conditions, icing of transmission line occurs widely in China and causes ice disasters accident such as short terms, pour towers [1]. For example, in January 2008, parts of Southern China suffered a rare sleet weather resulting in widespread icing of transmission lines, and ice thickness of some towers obviously went beyond line mechanical carrying capacity. The heavy ice cover even caused the collapse of towers, which not only strongly affected and threatened the safety and stability of the power grids but also caused huge economic loss[2].

In on-line monitoring systems, tension sensors and angle sensors are the bare essentials. Tension and angle of suspended points can reflect the role of the wind on conductors and the changes of conductors state. This paper detects the steady state icing of overhead power lines mainly through the combination of axial tension, deflecttion angel and swing angle of suspension point.

\section{Static Mechanics Analysis Model of the Straig- ht Tower}

In engineering calculations, they often overlook the rigidity of overhead conductors as flexible cable. And in fact, we assume that the wire load is evenly distributed along the deflection span, for the reason that the difference between the arc length of the wire and the distance of the two suspension points in a span is very small. For the above reasons,the inclined parabola formulas can be applied in the calculation of the wires, and the error is within the allowable range in the engineering.[3]

\subsection{Statics Mechanics Analysis in the Vertical Plane}

The mechanical model of the overhead transmission lines in the condition of no wind and no ice is in Figure 1. Shown in the Figure 1, $l$ is the span length; $h$ is the height difference between the two neighboring towers; $\beta$ is the angle of height difference between the two neighboring suspension points; $l_{V}$ is the distance between the lowest points and the main tower; $\gamma$ is the load of the wire itself.

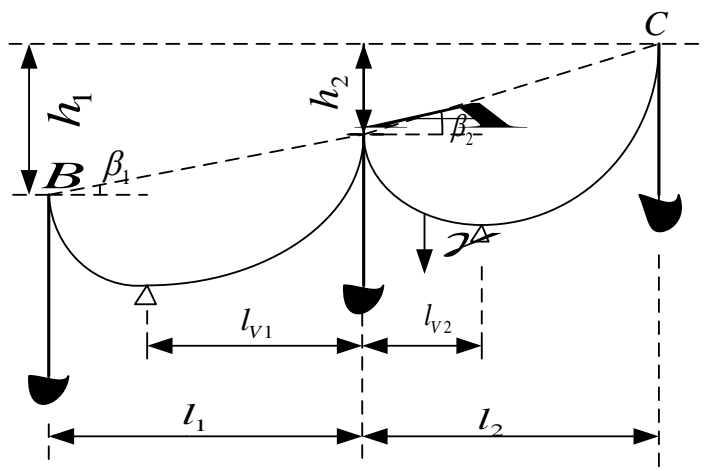

Figure 1.Transmission line model without external load in vertical plane of towers. 
By the horizontal force balance condition, we know that the horizontal component of each point tension are equal to the stress $\sigma_{o}$ of the lowest point. So

$$
F \cdot \cos \theta=\sigma_{0} A,
$$

where $F$ is the tension of the suspension points; $\theta$ is the deflection angle of the suspended points; $A$ is sectional area.

We have known $F, \theta$ and $A$, so we can calculate $\sigma_{o}$ through the equation(1).

1) the original length $S$ of wires in a span[3]

$$
S=\frac{l}{\cos \beta}+\frac{\gamma^{2} l^{3} \cos \beta}{24 \sigma_{0}{ }^{2}}
$$

2) the distance $l_{V}$ between the lowest points and the main tower[3]

$$
\begin{gathered}
l_{V 1}=\frac{l_{1}}{2}+\frac{\sigma_{o} h_{1}}{\gamma l_{1}} \\
l_{V 2}=\frac{l_{2}}{2}-\frac{\sigma_{0} h_{2}}{\gamma l_{2}}
\end{gathered}
$$

3) the wire length $S_{V}$ between the lowest points and the main tower[4]

$$
\begin{gathered}
S_{V 1}=l_{V 1}+\frac{\gamma^{2} l_{V 1}^{3}}{6 \sigma_{o}{ }^{2} \cos ^{2} \beta_{1}} \\
S_{V 2}=l_{V 2}+\frac{\gamma^{2} l_{V 2}{ }^{3}}{6 \sigma_{o}{ }^{2} \cos ^{2} \beta_{2}}
\end{gathered}
$$

\subsection{The Mechanics Analysis in Windage Yaw Plane}

In engineering calculation, the wire length is approximately equal to the inclined span length, so the angle between the wind and the wire can be treated as the angle between the wind and the inclined span approximately. Under this assumption, and by the effect of wind, the overhead transmission lines must lie in the plane formed by the line of the comprehensive load action.[3]

In the existing model, the mechanical calculation of the icing wires is only in the vertical plane without consider- ing the influence of wind. But the tension sensors can only detect the magnitude of force and can't measure the direction. In fact, the tension is not fixed in the vertical plane, and it will change as the change of the deflection of the wires due to the wind. In this paper, the mechanical analysis is in the new coordinate plane due to wind.

As is shown in Figure 2, the parameters' relation of the vertical plane and the windage yaw plane is this[3]:

$$
\begin{gathered}
l^{\prime}=l \sqrt{1+(\tan \beta \sin \eta)^{2}} \\
h^{\prime}=h \cos \eta
\end{gathered}
$$

$$
\begin{gathered}
\sigma_{o}^{\prime}=\sigma_{o} \sqrt{1+(\tan \beta \sin \eta)^{2}} \\
\cos \beta^{\prime}=\cos \beta \sqrt{1+(\tan \beta \sin \eta)^{2}} \\
\sin \beta^{\prime}=\sin \beta \cos \eta \\
\sigma_{A}^{\prime}=\frac{\sigma_{o}}{\cos \beta}+\gamma^{\prime}\left(\frac{\gamma^{\prime} l^{2}}{8 \sigma_{o} \cos \beta}-\frac{h \cos \eta}{2}\right) \\
\sigma_{B}^{\prime}=\frac{\sigma_{o}}{\cos \beta}+\gamma^{\prime}\left(\frac{\gamma^{\prime} l^{2}}{8 \sigma_{o} \cos \beta}+\frac{h \cos \eta}{2}\right)
\end{gathered}
$$

where $l^{\prime}$ is the span; $h^{\prime}$ is the height difference; $\beta^{\prime}$ is the angle of height difference; $\sigma_{A}^{\prime}$ is the horizontal stress; $\sigma_{A}^{\prime}, \sigma_{B}^{\prime}$ is respectively the tension of the suspended points $A$ and $B$. What's more,all the above parameters are in the windage yaw plane. And $\sigma_{o}$ is the horizontal stress in the vertical projective plane, and $\eta$ is the windage yaw angle.

Angle sensors usually detect the deflection angle of the suspended points in the vertical plane. And the equation (14) is shown: [3]

$$
\tan \theta_{V A}=\tan \beta+\gamma_{V} l /\left(2 \sigma_{o} \cos \beta\right)
$$

The relationship between the vertical comprehensive load $\gamma^{\prime}$ in windage yaw plane and the vertical load $\gamma_{V}$ is that:

$$
\gamma^{\prime}=\gamma_{V} / \cos \eta
$$

Take advantage of the wire axial tension $F$ of the point $A$ measured by the tension sensor, windage yaw angle $\eta$ measured by the angle sensor and the deflection angle $\theta_{V A}$ of suspended point $A$ in the vertical plane, we can work out the comprehensive load $\gamma^{\prime}$ of the wire and the horizontal tension $\sigma_{o}^{\prime}$ of the wire in vertical plane accordi- ng to Equations (12)(14)(15).

According to the Equations (2)(5)(6), in the windage yaw plane, the length $S^{\prime}$ of the wire in a span and the lengths $S_{V 1}^{\prime}$ and $S_{V 2}^{\prime}$ of the wire between the lowest point and the main towerare are as follows:

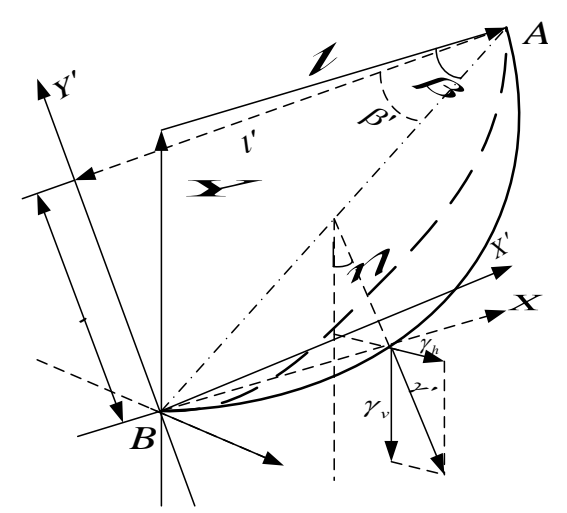

Figure 2. The mechanics analysis of the conductor in windage yaw plane. 


$$
\begin{aligned}
& S^{\prime}=\frac{l^{\prime}}{\cos \beta^{\prime}}+\frac{\gamma^{\prime 2} l^{\prime 3} \cos \beta^{\prime}}{24 \sigma_{o}^{\prime 2}} \\
& S_{V 1}^{\prime}=l_{V 1}^{\prime}+\frac{\gamma^{\prime 2} l_{V 1}^{\prime 3}}{6 \sigma_{o}^{\prime 2} \cos \beta_{1}^{\prime 2}} \\
& S_{V 2}^{\prime}=l_{V 2}^{\prime}+\frac{\gamma^{\prime 2} l_{V 2}^{\prime 3}}{6 \sigma_{o}^{\prime 2} \cos \beta_{2}^{\prime 2}}
\end{aligned}
$$

\subsection{The Mechanics Analysis of Icing Wires in the New Coordinate Plane Due To Wind}

Because of the change of meteorological conditions, the conductor tensions in different spans will change by the parameters of themselves. And it will result that wires of different spans have different horizontal tensions, and the suspended points will occur horizontal movement until the horizontal tensions of the neighbouring spans are equal. The model of the icing wires in the windage yaw plane is shown as Figure 3, and the position of insulators and wires is described in Figure 4.

In order to simplify the calculation, we assume that the deflection angle of insulator chain is the same to the suspended point's. And considering that the difference of $\theta^{\prime}$ and $\theta_{A}^{\prime}$ is small, we assume that $\theta^{\prime} \approx \theta_{A}^{\prime}$ and $\theta_{V}^{\prime} \approx \theta_{V A}^{\prime}$.

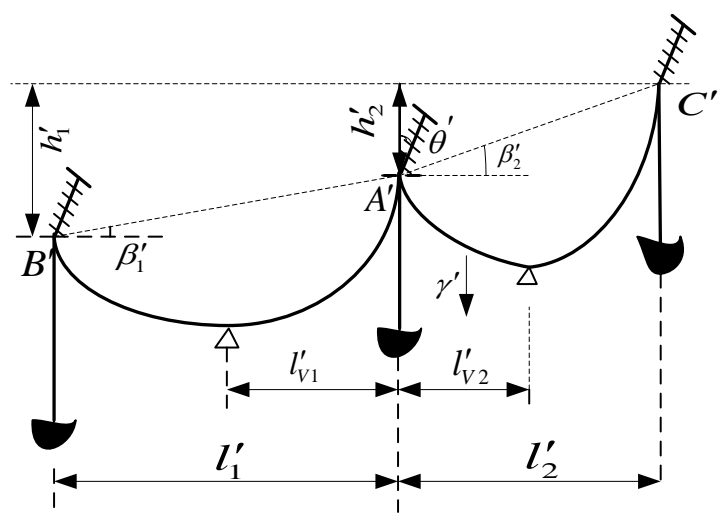

Figure 3. The calculating model of icing wires in the windage yaw plane.

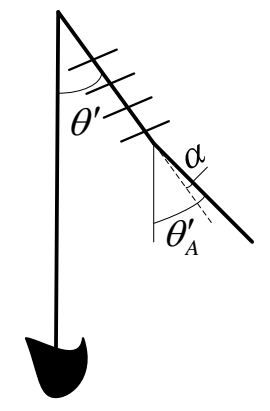

Figure 4. The system of insulator-wire in the new coordinate plane due to wind.
According to the above hypothesis, the deflection angle $\theta^{\prime}$ and the windage yaw angle $\eta$ and the deflection angle $\theta_{V A}$ of the suspended point $A$ in the vertical plane have this relationship[5]:

$$
\cos \theta^{\prime}=1 /\left(\cos \eta \sqrt{1+\tan ^{2} \eta+\tan ^{2} \theta_{V A}}\right)
$$

For the suspended point $A$, the equation about the vertical load $\gamma_{V}$ and the vertical component of the tension $F_{A}$ is shown as:

$$
F_{\mathrm{A}} \cos \theta^{\prime}=\left[\gamma_{V} \mathrm{~A}\left(\mathrm{~S}_{\mathrm{V} 1}^{\prime}+\mathrm{S}_{\mathrm{V} 2}^{\prime}\right)\right] / \cos \eta
$$

where $\gamma_{V}$ is the comprehensive vertical load of the icing wires, and $\gamma_{V}=\gamma+\gamma_{\text {ice }} ; \gamma_{\text {ice }}$ is the load of the icing wires.

The equation (20) can deduce the load of icing wire:

$$
\gamma_{\text {ice }}=\frac{F_{A} \cos \theta^{\prime} \cos \eta}{\left(S_{V 1}^{\prime}+S_{V 2}^{\prime}\right) A}-\gamma
$$

According to the calculation icing load and the shape of icing conductor which is set to a uniform cylindrical by the transmission line design standard of power system, then the calculation formula of icing thickness is as follows:

$$
b=0.5\left(\sqrt{\frac{4 \gamma_{\text {ice }} A}{9.8 \pi \rho}+d^{2}}-d\right)
$$

where $d$ is the calculation diameter of conductor; $b$ is the icing thickness; $\rho$ is the icing density, and it's usually equal to $0.9 \mathrm{~g} / \mathrm{m}^{3}$.

\section{Demonstration}

We will verify the validity of this model by calculating the wind load $\gamma_{\text {wind }}$ of the icing wire [6].

It's called the wind load $\gamma_{\text {wind }}$ of icing wires that icing wires of per meter and per square millimeter withstand wind press load.The expression of $\gamma_{\text {wind }}$ algorithm is as follows:

$$
\gamma_{\text {wind }}=\frac{0.6125 \alpha C(2 b+d) v^{2}}{A} \times 10^{-3}
$$

where $\alpha$ is the non-uniformn coefficient of wind speed, the values are shown in Table 1; $v$ is the design wind speed; $C$ is the figure coefficient of wind load, and it's usually equal to 1.2. If the ratio of $\gamma_{\text {wind }}$ and $\gamma_{V}$ is equal to $\tan \eta$, then the model in this paper is validity.

Table 1. The value of in different conditions

\begin{tabular}{ccccc}
\hline$v(\mathrm{~m} / \mathrm{s})$ & $\leq 20$ & $20 \sim 30$ & $30 \sim 35$ & $\geq 35$ \\
\hline$\alpha$ & 1.0 & 0.85 & 0.75 & 0.70 \\
\hline
\end{tabular}




\section{Conclusions}

In this paper, we just build a model in theory that applicable to the usual on-line monitoring. As for as the accuracy and practicability, practical examples have yet to prove the model. If the model is correct, it will be applicable to the conditions that monitoring systems have not image detection or that the weather is freezing and affects the icing monitoring.

The new model can get icing information, so that we can timely de-icing if the icing is beyond line mechanical carrying capacity and guarantee the normal use of electric transmission wires in the freezing climate.

\section{REFERENCES}

[1] X. L. Jiang and H. Yi, "Transmission Line Icing and Protection,” China Electric Power Press, Beijing, 2002.

[2] H. Hou, X. G. Yin, Q. Q. Chen, et al., "Review on the
Wide Area Blackout of $500 \mathrm{kV}$ Main Power Grid in Some Areas of South China in 2008 Snow Disaster," Automation of Electric Power Systems, Vol. 32, No. 8, 2008, pp. 12-15.

[3] X. T. Shao, “The Wire's Mechanical Calculion of Overhead Transmission Line,” China Water Power Press, Beijing, 2003.

[4] B. Z. Li, "Stringing Technique Calculation Principle of High Voltage Overhead Transmission Line,” China Electric Power Press, Beijing, 2002.

[5] L. Yang, Y. P. Hao, W. G. Li, D. Dai, L. C. Li, G. H. Zhu and B. Luo, “A Mechanical Calculation Model for On-line Icing-monitoring System of Overhead Transmission Lines,” Proceedings of the CSEE, Vol. 30, No.19, 2010, pp.100-105.

[6] Y. X. Lu and Y. Zhou, "Simple Models for Calculating Weight of Ice Accretion on Transmission Lines," East China Electric Power, Vol. 37, No. 3, 2009, pp. 0433-0435. 